\title{
A comparison of patient pain and visual outcome using topical anesthesia versus regional anesthesia during cataract surgery
}

This article was published in the following Dove Press journal:

Clinical Ophthalmology

20 June 2016

Number of times this article has been viewed

\section{Yoshikatsu Hosoda \\ Shoji Kuriyama \\ Yoko Jingami \\ Hidetsugu Hattori \\ Hisako Hayashi \\ Miho Matsumoto}

Department of Ophthalmology, Otsu Red-Cross Hospital, Otsu, Japan
Correspondence: Yoshikatsu Hosoda Department of Ophthalmology, Otsu Red-Cross Hospital, I-I-35 Nagara, Otsu, Shiga 520-0046, Japan

Tel +8I $775224|3|$

Email kubrick@kuhp.kyoto-u.ac.jp
Purpose: The purpose of this study was to compare the level of patient pain during the phacoemulsification and implantation of foldable intraocular lenses while under topical, intracameral, or sub-Tenon lidocaine.

Patients and methods: This was a retrospective study. Three hundred and one eyes subjected to cataract surgery were included in this study. All eyes underwent phacoemulsification surgery and intraocular lens implantation using topical, sub-Tenon, or intracameral anesthesia. The topical group received $4 \%$ lidocaine drops, and the intracameral group received a $0.1-0.2$ cc infusion of $1 \%$ preservative-free lidocaine into the anterior chamber through the side port combined with topical drops of lidocaine. The sub-Tenon group received $2 \%$ lidocaine. Best-corrected visual acuity, corneal endothelial cell loss, and intraoperative pain level were evaluated. Pain level was assessed on a visual analog scale (range $0-2$ ).

Results: There were no significant differences in visual outcome and corneal endothelial cell loss between the three groups. The mean pain score in the sub-Tenon group was significantly lower than that in the topical and intracameral groups ( $P=0.0009$ and $P=0.0055$, respectively). In 250 eyes without high myopia $(<-6 \mathrm{D})$, there were no significant differences in mean pain score between the sub-Tenon and intracameral groups $(P=0.1417)$. No additional anesthesia was required in all groups.

Conclusion: Intracameral lidocaine provides sufficient pain suppressive effects in eyes without high myopia, while sub-Tenon anesthesia is better for cataract surgery in eyes with high myopia.

Keywords: cataract, anesthesia, topical, intracameral, sub-Tenon

\section{Background}

Phacoemulsification with intraocular lens implantation is the current standard of care for adult cataract surgery. Several methods of local anesthesia for cataract extraction are currently available. These include administration of retrobulbar, ${ }^{1,2}$ peribulbar, ${ }^{3-5}$ subconjunctival, ${ }^{6}$ sub-Tenon, ${ }^{7}$ and topical anesthetic drops. ${ }^{6,8}$ Topical anesthesia is a reasonable alternative method to traditional local anesthesia injection, because potentially serious complications associated with retrobulbar or peribulbar anesthesia can be avoided. Previous studies reported sufficient pain repression only with topical anesthesia during cataract surgery. ${ }^{6,8}$

Sub-Tenon anesthesia, also known as parabulbar anesthesia, is a popular alternative to peribulbar or retrobulbar anesthesia. The sub-Tenon space is the potential space between the capsule and the sclera. Local anesthetics injected into this space lead to immediate analgesia and akinesia of the eyeball. Recent studies regarding local anesthesia 
techniques for cataract surgery found that sub-Tenon anesthesia results in less pain compared with topical anesthesia. ${ }^{9,10}$

Intracameral anesthesia is one of the most popular methods used in cataract surgery. ${ }^{11}$ The procedure involves the injection of anesthetics directly into the anterior chamber of the eye at the start of the surgery. The procedure has gained popularity recently, with lidocaine being the most common drug used for this purpose. The safety and efficacy when combined with topical anesthesia have been reported in various previous studies. ${ }^{12-16}$ Patients who might benefit from intracameral anesthesia include those with high myopia. The thinly stretched Zinn's zonules and the underdeveloped ciliary body of the highly myopic eye often lead to posterior displacement of the lens-iris diaphragm during anterior chamber infusion. This causes severe deepening of the anterior chamber, posterior iris bowing, and pupil dilation, which can cause pain during cataract surgery. ${ }^{17}$

In the literature comparing topical anesthesia and intracameral anesthesia, there are contradictory outcomes and conclusions. ${ }^{18}$ Tseng and $\mathrm{Chen}^{13}$ reported that combining topical and intracameral lidocaine administration can minimize intraoperative discomfort compared with topical anesthesia alone. However, other studies reported no significant differences in pain control between intracameral anesthesia and topical anesthesia. Mustapha et al ${ }^{19}$ reported that intracameral anesthesia obviates the need for an injection during cataract surgery and is as comfortable for patients as sub-Tenon anesthesia in terms of visual perception, level of pain, and anxiety.

However, the comparison of anxiety and visual outcome between the three types of anesthesia has not been reported in previous studies. The aim of this study was to compare the level of pain experienced during phacoemulsification and intraocular lens implantation in patients receiving either topical, intracameral, or sub-Tenon anesthesia.

\section{Patients and methods}

\section{Patients and ophthalmic examinations}

This is a retrospective, observational study. This study adhered to the tenets of the Declaration of Helsinki, and its protocol was approved by the Institutional Review Board of Otsu Red Cross Hospital. Consecutive patients who underwent phacoemulsification surgery and intraocular lens implantation at Otsu Red Cross Hospital from February 2015 to May 2015 were included in this study. Patients with a history of vitrectomy were excluded from this study. Prior to the surgery, all patients underwent comprehensive ophthalmologic evaluations. This included standardized refraction and measurement of best-corrected visual acuity (BCVA) using a Landolt ring, slit-lamp biomicroscopy, and specular microscopy. Written informed consent was obtained from every patient participating in this study.

All the groups underwent identical preoperative preparation with $0.4 \%$ oxybuprocaine hydrochloride, $4 \%$ lidocaine, tropicamide, and phenylephrine hydrochloride 60 minutes prior to surgery. Six trained ophthalmologists performed all the surgical procedures, including the administration of local anesthesia. The topical group received drops of $4 \%$ lidocaine instilled at the start of surgery. The anesthetic agent used was $1 \mathrm{~mL}$ of $2 \%$ intravenous lidocaine for the sub-Tenon group, and a purpose-designed sub-Tenon's cannula was used to instill the agents.

Patients in the intracameral group also received drops of $4 \%$ lidocaine instilled at the start of surgery. At the start of the surgery, an infusion of $0.1-0.2 \mathrm{cc}$ of $1 \%$ preservativefree lidocaine was infused into the anterior chamber through the side port before the chamber was filled with viscoelastic materials. No general or preoperative sedation or other anesthesia was used in all groups.

All patients underwent surgery using the same technique. A $2.2 \mathrm{~mm}$ groove was made in the sclera or clear cornea. Continuous curvilinear capsulorhexis with capsule forceps was performed followed by hydrodissection of the lens with balanced salt resolution. All patients received a foldable acrylic lens. No wound sutures were required for any of the patients. Complicated surgeries, such as posterior capsule rupture or zonular dialysis, were excluded from this study. In cases where patients claimed unbearable pain, an additional sub-Tenon injection of $2 \%$ lidocaine was administered.

The patients were asked within 5 minutes after the surgery to grade the pain experienced during surgery on an analog scale. Pain was graded on a linear scale from 0 to 2 (grade $0=$ no pain, grade $1=$ mild pain, and grade $2=$ severe pain). Independent nurses asked the patients to provide a pain score in a separate room. The patients were followed up for at least 3-4 weeks after the surgery, and corneal endothelial damage and BCVA were evaluated. The statistical differences in pain score, visual outcome, and corneal endothelial damage between the three groups were evaluated.

\section{Statistical analyses}

Data are expressed as mean \pm standard deviation. All BCVA measurements were converted to logarithm of the minimum angle of resolution equivalents for statistical analyses. Analysis of variance and Tukey-Kramer method were used to compare ophthalmic data between the three groups (topical, sub-Tenon, 
Table I Baseline demographics and ophthalmic examination data of patients who underwent cataract surgery

\begin{tabular}{|c|c|c|c|c|}
\hline & Topical & Sub-Tenon & Intracameral & $P$-value \\
\hline Eyes, $\mathrm{n}$ & 101 & 101 & 99 & - \\
\hline Male/female, $\mathrm{n}$ & $42 / 59$ & $51 / 50$ & $44 / 55$ & - \\
\hline Age (years), mean $\pm S D$ & $74.5 \pm 7.3$ & $71.5 \pm 9.5$ & $72.5 \pm 8.9$ & 0.0427 \\
\hline BCVA before surgery (LogMAR), mean \pm SD & $0.232 \pm 0.205$ & $0.363 \pm 0.463$ & $0.272 \pm 0.311$ & 0.0223 \\
\hline BCVA after surgery (LogMAR), mean \pm SD & $-0.023 \pm 0.135$ & $0.040 \pm 0.321$ & $0.008 \pm 0.221$ & 0.1700 \\
\hline Mean operative time $(\mathrm{min})$ & 8.8 & 9.8 & 9.2 & 0.0163 \\
\hline \multicolumn{5}{|l|}{ Pain score, $\mathrm{n}$} \\
\hline Grade 0 & 46 & 73 & 49 & - \\
\hline Grade I & 52 & 26 & 47 & - \\
\hline Grade 2 & 3 & 2 & 3 & - \\
\hline Mean pain score & 0.574 & 0.297 & 0.535 & 0.0004 \\
\hline \multicolumn{5}{|l|}{ Mean endothelial cell count (cells $/ \mathrm{mm}^{2}$ ) } \\
\hline Preoperative & 2,606 & 2,578 & 2,632 & 0.4870 \\
\hline Postoperative & 2,414 & 2,376 & 2,384 & 0.8370 \\
\hline Mean cell loss (\%) & 7.7 & 7.7 & 8.5 & 0.9430 \\
\hline
\end{tabular}

Abbreviations: SD, standard deviation; BCVA, best-corrected visual acuity; LogMAR, logarithm of the minimum angle of resolution; min, minutes.

and intracameral anesthesia). Significance was defined as $P<0.05$, and all statistical analyses were performed using R software (Version 3.1.3; R Foundation, Vienna, Austria).

\section{Results}

Three hundred six eyes were included in this study. Five eyes were excluded: four eyes were excluded due to posterior capsule rupture, while the remaining eye was excluded due to a broken intraocular lens during surgery. In total, 301 eyes in 190 patients were included in this study. One hundred one patients (mean age $74.5 \pm 7.3$ years) received topical anesthesia, 101 patients (mean age $71.5 \pm 9.5$ years) received sub-Tenon anesthesia, and 99 patients (mean age $72.5 \pm 8.9$ years) received intracameral anesthesia. None of the patients required additional anesthesia during the surgery. There was a significant difference in mean age between the topical group and sub-Tenon group ( $P=0.0377$; Table 1).

Additionally, the baseline BCVA in the topical group was significantly better than that in the sub-Tenon group $(P=0.0195)$. However, there were no significant differences in visual outcome after the surgery between the three groups. Table 1 shows the mean corneal endothelial cell count. The reduction in endothelial cell count was $7.7 \%$ in the topical anesthesia group, $7.7 \%$ in the sub-Tenon group, and $8.5 \%$ in the intracameral anesthesia group. The change in endothelial cell density was not significantly different between the three groups. The mean pain score on the visual pain scale in the sub-Tenon group was significantly lower than that in the topical group $(P=0.0009)$ and in the intracameral group $(P=0.0055)$. There were no statistical differences in mean pain score between the topical and intracameral groups $(P=0.8661)$.

In our study, 111 patients received bilateral cataract surgery and 79 patients received unilateral surgery. The mean pain score was 0.405 in 79 eyes of 79 patients who received bilateral surgery and 0.491 in 222 eyes of 111 patients who received unilateral surgery. There was no significant difference in mean pain score between the two groups $(P=0.2342)$.

Table 2 shows the characteristics and pain score of 190 first eyes that underwent cataract surgery. A subgroup analysis showed that the mean pain score in the sub-Tenon

Table 2 Ophthalmic examination data of first eyes of patients who underwent cataract surgery

\begin{tabular}{lllll}
\hline & Topical & Sub-Tenon & Intracameral & P-value \\
\hline Eyes, $\mathrm{n}$ & 68 & 66 & 56 & - \\
Age (years), mean \pm SD & $74.5 \pm 7.3$ & $71.2 \pm 9.6$ & $72.3 \pm 9.6$ & 0.0427 \\
BCVA before surgery (LogMAR), mean \pm SD & $0.260 \pm 0.212$ & $0.403 \pm 0.487$ & $0.348 \pm 0.373$ & 0.0865 \\
BCVA after surgery (LogMAR), mean \pm SD & $-0.016 \pm 0.148$ & $0.006 \pm 0.268$ & $0.034 \pm 0.274$ \\
Pain score, $\mathrm{n}$ & & & & 0.5010 \\
Grade 0 & 34 & 47 & 29 & - \\
Grade I & 31 & 19 & 26 & - \\
Grade 2 & 3 & 0 & 1 & - \\
Mean pain score & 0.544 & 0.288 & 0.500 & 0.0136 \\
\hline
\end{tabular}

Abbreviations: SD, standard deviation; BCVA, best-corrected visual acuity; LogMAR, logarithm of the minimum angle of resolution. 
Table 3 Detailed characteristics of 190 first eyes of patients

\begin{tabular}{llll}
\hline & Bilateral & Unilateral & P-value \\
\hline Eyes, $\mathrm{n}$ & 79 & $\mathrm{III}$ & - \\
Age (years), mean \pm SD & $72.3 \pm 9.5$ & $73.0 \pm 8.4$ & 0.5858 \\
$\begin{array}{l}\text { BCVA before surgery } \\
\text { (LogMAR), mean } \pm \text { SD }\end{array}$ & $0.268 \pm 0.326$ & $0.384 \pm 0.400$ & 0.0362 \\
$\begin{array}{l}\text { BCVA after surgery } \\
\text { (LogMAR), mean } \pm \text { SD }\end{array}$ & $-0.034 \pm 0.198$ & $0.035 \pm 0.25 \mathrm{I}$ & 0.0434 \\
Anesthesia, $\mathrm{n}$ & & & \\
$\quad$ & & & \\
$\quad$ Topical & 27 & $4 \mathrm{I}$ & - \\
$\quad$ Intracameral & 30 & 36 & - \\
$\quad$ Mean pain score & 22 & 34 & - \\
\hline
\end{tabular}

Abbreviations: SD, standard deviation; BCVA, best-corrected visual acuity; LogMAR, logarithm of the minimum angle of resolution.

group was significantly lower than that in the topical group $(P=0.0155)$. The intracameral anesthesia group showed no statistically significant difference compared with sub-Tenon and topical anesthesia groups $(P=0.0728$ and $P=0.8892$, respectively). In 111 first eyes of 111 patients who received unilateral surgery, the mean pain score was 0.468 (Table 3 ). There was no significant difference in mean pain score between the bilateral and unilateral groups $(P=0.4255)$.

Figure 1 shows the average of pain scores in patients with and without high myopia. Fifty-one eyes in 30 patients showed high myopia ( $>-6 \mathrm{D}$ ). In 51 myopic eyes, there were no significant differences in age, duration of surgery, visual acuity, and mean percentage of corneal endothelial cell loss between the three groups (topical, sub-Tenon, and intracameral). The mean pain score in the sub-Tenon group was significantly lower than that in the topical and intracameral groups ( $P=0.0031$ and $P=0.0020$, respectively). However, in 250 eyes without high myopia $(<-6 \mathrm{D})$, there were no significant differences in mean pain score between the subTenon and intracameral groups $(P=0.1417)$. There were no significant differences in baseline characteristics and visual outcome between the three groups.

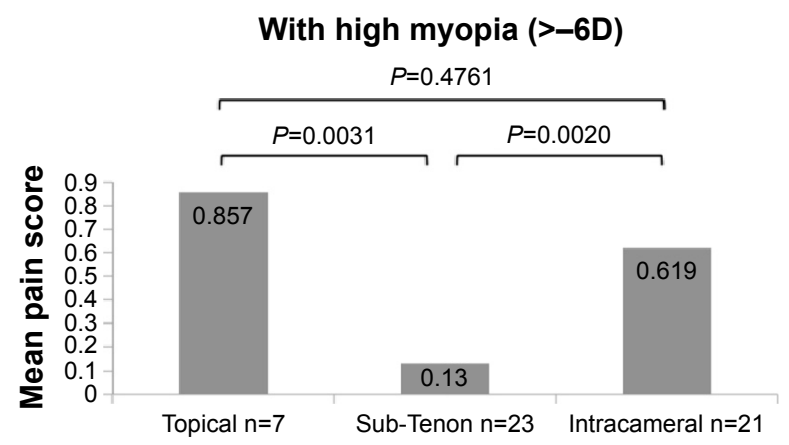

Six surgeons performed all the operations, and the individual outcomes of mean pain score are shown in Table 4. There were no significant differences in mean pain score between the six operators.

\section{Discussion}

To the best of our knowledge, this is the first study to compare the efficacy of three types of anesthesia (topical, sub-Tenon, and intracameral) during cataract surgery. In the present study, all types of anesthesia provided good patient comfort, as none of the patients required additional anesthesia during surgery. Additionally, no complications caused by anesthesia were noted in any of the patients. There were no differences in visual outcome and corneal endothelial cell loss between the groups. The mean percentage of corneal endothelial loss in all patients was $8.0 \%$, and this result is almost similar to previous studies. ${ }^{3,18}$ The duration of surgery was shortest in the topical group; this may be because the anesthetic procedure is simpler and easier than sub-Tenon and intracameral anesthesia.

Several studies have reported almost no differences in mean pain scores between intracameral lidocaine and placebo in patients undergoing cataract surgery under topical anesthesia. ${ }^{8,12,17}$ Most statistical analyses did not yield strong data supporting the efficacy of additional intracameral anesthesia during phacoemulsification under topical anesthesia. However, Ezra et $\mathrm{al}^{20}$ reported a statistically significant benefit from the use of this technique. In our study, there were no statistically significant differences in pain scores during surgery between the topical and intracameral lidocaine groups (mean pain score 0.574 and 0.535 , respectively). Pain scores using both anesthesia techniques were consistently low; $>90 \%$ of patients scored pain as grade 1 or 0 . It is possible that topical anesthesia alone is sufficiently effective and the additional benefit with intracameral anesthesia is minimal.

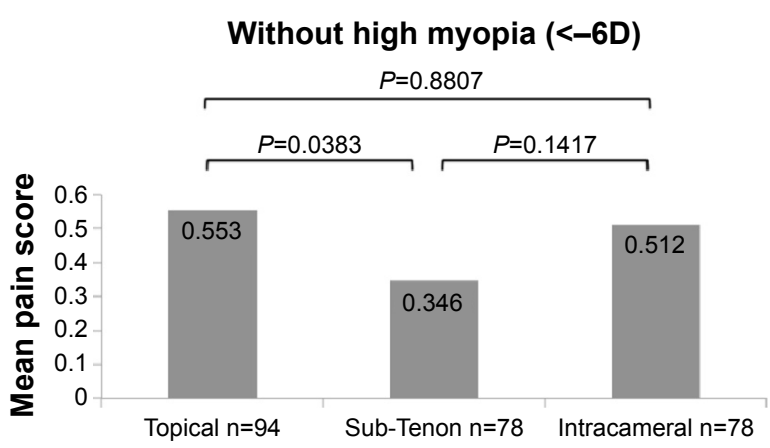

Figure I The mean pain scores during cataract surgery in patients with and without high myopia. 
Table 4 Individual pain score of eyes operated by six surgeons (SK, MM, H Hattori, H Hayshi, YJ, YH)

\begin{tabular}{|c|c|c|c|c|c|c|c|}
\hline & SK & MM & H Hattori & H Hayshi & YJ & $\mathbf{Y H}$ & $P$-value \\
\hline \multicolumn{8}{|l|}{ Topical group } \\
\hline Eyes, $\mathrm{n}$ & 19 & 10 & 24 & 14 & 16 & 18 & \\
\hline Mean pain score & 0.368 & 0.600 & 0.625 & 0.714 & 0.500 & 0.667 & 0.484 \\
\hline \multicolumn{8}{|l|}{ Sub-Tenon group } \\
\hline Eyes, $\mathrm{n}$ & 19 & II & 24 & 14 & 15 & 18 & \\
\hline Mean pain score & 0.105 & 0.454 & 0.250 & $0.57 I$ & 0.267 & 0.278 & 0.134 \\
\hline \multicolumn{8}{|l|}{ Intracameral group } \\
\hline Eyes, $\mathrm{n}$ & 18 & 10 & 23 & 14 & 15 & 19 & \\
\hline Mean pain score & 0.444 & 0.700 & 0.565 & 0.643 & 0.533 & 0.421 & 0.752 \\
\hline \multicolumn{8}{|l|}{ Total } \\
\hline Eyes, $\mathrm{n}$ & 56 & 31 & 71 & 42 & 46 & 55 & \\
\hline Mean pain score & 0.304 & 0.581 & 0.479 & 0.643 & 0.435 & 0.455 & 0.055 \\
\hline
\end{tabular}

Our findings indicate that sub-Tenon anesthesia in cataract surgery can suppress patient pain better than topical and intracameral anesthesia. The patients in the sub-Tenon group showed remarkable results in terms of the apparent painlessness of the surgery. Almost no pain was reported in 73 out of 101 eyes $(72.3 \%)$ during surgery. In particular, in eyes with high myopia, sub-Tenon anesthesia provided reasonable suppression of intraoperative pain compared with topical and intracameral anesthesia. This result suggests that intracameral lidocaine does not provide sufficient pain suppression in eyes with high myopia because of the thinly stretched Zinn's zonules and the underdeveloped ciliary body. However, in eyes without high myopia, there were no significant differences in mean pain score between subTenon and intracameral anesthesia. Intracameral lidocaine combined with topical anesthesia was not inferior to subTenon anesthesia in regular refraction eyes.

In the present study, 111 patients received bilateral cataract surgery and 79 patients received unilateral surgery. Roxana et $\mathrm{al}^{21}$ reported that there was a subtle increase in pain during cataract surgery in the second eye relative to the first. The result of our subgroup analysis restricted to first 190 eyes also showed no significant differences in mean pain score between sub-Tenon and intracameral anesthesia. Additionally, there was no significant difference in mean pain score between the two groups who received bilateral or unilateral surgery.

This study has several limitations. We evaluated pain only during surgery; the pain during instillation of anesthesia and anxiety from visual light should be evaluated separately. It is possible that patients included the pain on injection of anesthetic agents in the pain score. In previous studies, ${ }^{13}$ balanced salt solution was used to compare local and topical anesthesia. Using a placebo may be preferable in order to obtain an accurate comparison of anesthetic effect.
In our study, six independent surgeons performed all surgical procedures, and the kinds of grooves, in the sclera or cornea, and the types of intraocular lenses depend on the surgeon's preferences. However, there were no significant differences in mean pain score between the six operators. This result means that the kinds of grooves or intraocular lenses have no significant effect on the pain score.

\section{Conclusion}

Intracameral lidocaine for cataract surgery shows sufficient pain suppressive effects in eyes without high myopia. Based on the results from this study, we believe that sub-Tenon anesthesia is better for cataract surgery in eyes with high myopia.

\section{Acknowledgment}

This study did not receive any financial support.

\section{Disclosure}

The authors report no conflicts of interest in this work.

\section{References}

1. Ellis P. Retrobulbar injections. Surv Ophthalmol. 1974;18:425-430.

2. Patel BC, Burns TA, Crandall A, et al. A comparison of topical and retrobulbar anesthesia for cataract surgery. Ophthalmology. 1996;103(8): 1196-1203.

3. Arnold PN. Prospective study of a single-injection peribulbar technique. $J$ Cataract Refract Surg. 1992;18(2):157-161.

4. Zhao L-Q, Zhu H, Zhao P-Q, Wu Q-R, Hu Y-Q. Topical anesthesia versus regional anesthesia for cataract surgery: a meta-analysis of randomized controlled trials. Ophthalmology. 2012;119(4):659-667.

5. Zehetmayer M, Radax U, Skorpik C, et al. Topical versus peribulbar anesthesia in clear corneal cataract surgery. J Cataract Refract Surg. 1996; 22(4):480-484.

6. Smith R. Cataract extraction without retrobulbar anaesthetic injection. Br J Ophthalmol. 1990;74(4):205-207.

7. Stevens J. A new local anesthesia technique for cataract extraction by one quadrant sub-Tenon's infiltration. Br J Ophthalmol. 1992;76(11): $670-674$. 
8. Pandey SK, Werner L, Apple DJ, Agarwal A, Agarwal A, Agarwal S. No-anesthesia clear corneal phacoemulsification versus topical and topical plus intracameral anesthesia: randomized clinical trial. J Cataract Refract Surg. 2001;27(10):1643-1650.

9. Rüschen H, Celaschi D, Bunce C, Carr C. Randomised controlled trial of sub-Tenon's block versus topical anaesthesia for cataract surgery: a comparison of patient satisfaction. Br J Ophthalmol. 2005;89(3):291-293.

10. Manners T, Burton R. Randomised trial of topical versus sub-Tenon's local anaesthesia for small-incision cataract surgery. Eye (Lond). 1996; 10(pt 3):367-370.

11. Koch PS. Anterior chamber irrigation with unpreserved lidocaine $1 \%$ for anesthesia during cataract surgery. J Cataract Refract Surg. 1997;23(4):551-554.

12. Crandall AS, Zabriskie NA, Patel BC, et al. A comparison of patient comfort during cataract surgery with topical anesthesia versus topical anesthesia and intracameral lidocaine. Ophthalmology. 1999; 106(1):60-66.

13. Tseng S-H, Chen FK. A randomized clinical trial of combined topical-intracameral anesthesia in cataract surgery. Ophthalmology. 1998;105(11):2007-2011.

14. Tan CS, Fam H-B, Heng W-J, Lee H-M, Saw S-M, Eong K-GA. Analgesic effect of supplemental intracameral lidocaine during phacoemulsification under topical anaesthesia: a randomised controlled trial. Br J Ophthalmol. 2011;95(6):837-841.
15. Westborg I, Mönestam E. Intracameral anesthesia for cataract surgery: a population-based study on patient satisfaction and outcome. Clin Ophthalmol. 2013;7:2063-2068.

16. Garcia A, Loureiro F, Limao A, Sampaio A, Ilharco J. Preservativefree lidocaine $1 \%$ anterior chamber irrigation as an adjunct to topical anesthesia. J Cataract Refract Surg. 1998;24(3):403-406.

17. Lofoco G, Ciucci F, Bardocci A, et al. Efficacy of topical plus intracameral anesthesia for cataract surgery in high myopia: randomized controlled trial. J Cataract Refract Surg. 2008;34(10):1664-1668.

18. Chuang L-H, Yeung L, Ku W-C, Yang K-J, Lai C-C. Safety and efficacy of topical anesthesia combined with a lower concentration of intracameral lidocaine in phacoemulsification: paired human eye study. J Cataract Refract Surg. 2007;33(2):293-296.

19. Mustapha M, Hamzah MH, Chow SK, et al. Sub-tenon anaesthesia versus intracameral anaesthesia in patients undergoing cataract extraction: a comparative study of the level of pain, visual perception and anxiety. Int J Med Stud. 2014;2(2):49-52.

20. Ezra DG, Nambiar A, Allan BD. Supplementary intracameral lidocaine for phacoemulsification under topical anesthesia: a meta-analysis of randomized controlled trials. Ophthalmology. 2008;115(3):455-487.

21. Roxana U, Matthew TF, Michael Z, et al. Pain perception in sequential cataract surgery: Comparison of first and second procedures. J Cataract Refract Surg. 2011;37(6):1009-1014.
Clinical Ophthalmology

\section{Publish your work in this journal}

Clinical Ophthalmology is an international, peer-reviewed journal covering all subspecialties within ophthalmology. Key topics include: Optometry; Visual science; Pharmacology and drug therapy in eye diseases; Basic Sciences; Primary and Secondary eye care; Patient Safety and Quality of Care Improvements. This journal is indexed on

\section{Dovepress}

PubMed Central and CAS, and is the official journal of The Society of Clinical Ophthalmology (SCO). The manuscript management system is completely online and includes a very quick and fair peer-review system, which is all easy to use. Visit http://www.dovepress.com/ testimonials.php to read real quotes from published authors. 